\title{
Generalization Lags behind Learning on an Auditory Perceptual Task
}

\author{
Beverly A. Wright, Roselyn M. Wilson, and Andrew T. Sabin \\ Department of Communication Sciences and Disorders and Institute for Neuroscience, Northwestern University, Evanston, Illinois 60208-3550
}

The generalization of learning from trained to untrained conditions is of great potential value because it markedly increases the efficacy of practice. In principle, generalization and the learning itself could arise from either the same or distinct neural changes. Here, we assessed these two possibilities in the realm of human perceptual learning by comparing the time course of improvement on a trained condition (learning) to that on an untrained condition (generalization) for an auditory temporal-interval discrimination task. While significant improvement on the trained condition occurred within $2 \mathrm{~d}$, generalization to the untrained condition lagged behind, only emerging after $4 \mathrm{~d}$. The different time courses for learning and generalization suggest that these two types of perceptual improvement can arise from at least partially distinct neural changes. The notably longer time course for generalization than learning demonstrates that increasing the duration of training can be an effective means to increase the number of conditions to which learning generalizes on perceptual tasks.

\section{Introduction}

Perceptual skills improve with practice, sometimes not just on the condition that was trained (learning) but also on untrained conditions (generalization). Such generalization is of key importance in practical terms because it maximizes the benefit received from training and in theoretical terms because its presence or absence is used to constrain learning theories. Yet, despite its significance, little is known about how the underlying changes that govern generalization to untrained perceptual conditions relate to those that govern learning on the trained condition. Here we provide behavioral evidence that the generalization of perceptual learning to an untrained condition can arise from neural changes that are at least partially distinct from those that yield the learning itself.

We assessed the relationship between the changes underlying perceptual learning and generalization by comparing the time courses of these two types of improvement. A difference in these two time courses would indicate that learning and generalization can arise from different changes. We are aware of only one previous direct comparison of these time courses in human perceptual learning. In that report, learning on a visual pop-out detection task generalized, at least in part, from a trained to an untrained stimulus orientation in participants who received one session of training (1400 trials) as well as in those who received 7-15 sessions (Ahissar and Hochstein, 1996). However, those data are of limited value in addressing the current question for two reasons. First, the observation of generalization both early and late in training does not preclude the possibility that the rate

Received March 20, 2010; revised June 22, 2010; accepted July 16, 2010.

This work was supported by the National Institute on Deafness and Other Communication Disorders/National Institutes of Health. We thank C. Aliyeva, R. Hu, J. Huyck, N. Marrone, J. Ortiz, and Y. Zhang for helpful comments on this manuscript.

Correspondence should be addressed to Beverly A. Wright, Department of Communication Sciences and Disorders, and Northwestern University Institute for Neuroscience, 2240 Campus Drive, Northwestern University, Evanston, IL 60208-3550. E-mail: b-wright@northwestern.edu.

D0I:10.1523/JNEUROSCI.1441-10.2010

Copyright $\odot 2010$ the authors $\quad$ 0270-6474/10/3011635-05\$15.00/0 of improvement for the trained and untrained conditions may have differed between the two time points tested. Second, even if the temporal trajectory was the same for both conditions, that correspondence could occur either because learning and generalization on that task derive from the same changes or derive from different changes that happen to have the same time course.

The results of the current investigation demonstrate that generalization can emerge more slowly than learning. We examined learning and generalization on an auditory temporal-interval discrimination task after 2,4 , and 10 daily sessions of training in three groups of listeners given these different amounts of practice. The key result is that the generalization of learning to an untrained stimulus frequency lagged behind the learning on the trained stimulus. Generalization appeared only well after improvements on the trained stimulus were evident. Thus, the generalization pattern broadened with increased training. This outcome suggests that perceptual learning and generalization can arise, in part, from different underlying changes. It also suggests that increasing the duration of training might be a means to increase the number of conditions to which learning generalizes on some perceptual tasks.

\section{Materials and Methods}

Generalization of stimulus learning. The generalization of learning on perceptual skills follows two primary patterns that are associated with different learning types (Ortiz and Wright, 2009). One pattern is characterized by generalization to all untrained conditions that share general aspects with the trained condition, such as the testing procedure or the particular judgment to be made, and therefore is attributed to the learning of conceptual components of the trained condition (conceptual learning). The other pattern is marked by generalization only to untrained conditions that employ stimuli that share particular features with the stimulus used during training, and thus reflects learning associated with the trained stimulus itself (stimulus learning). Of interest here is the generalization of stimulus learning (for an investigation of the relative time courses of stimulus and conceptual learning, see Ortiz and Wright, 2010).

Organization of experiment. The experiment consisted of a pretest, a training phase, and a posttest. During the pretest, we measured naive 


\section{Temporal-Interval Discrimination}

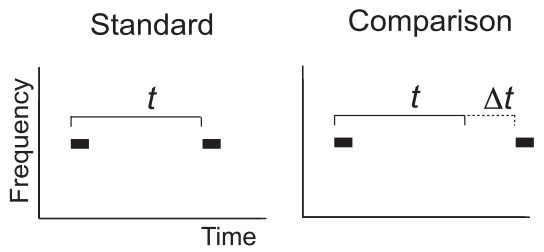

Figure 1. Temporal-interval discrimination task: on each trial, listeners were required to choose in which of two presentations the time between two brief tone pips was longer. The duration between the pips was always a standard value $(t)$ in one randomly selected presentation and a longer, comparison value in the other $(t+\Delta t)$. The $\Delta t$ at which listeners could discriminate the comparison from the standard stimulus on $79 \%$ of trials, termed "threshold," was estimated using an adaptive technique.

listeners' temporal-interval discrimination thresholds on a set of conditions, described below. During the training phase, listeners practiced one of the conditions from the pretest, the same one for all listeners. Different groups of listeners practiced for 2, 4, or $10 \mathrm{~d}$. Finally, during the posttest, we retested all listeners on the same conditions as the pretest. For all three groups, the pretest and first day of training were on consecutive days, as were the last day of training and the posttest. The intervening days of training typically occurred on consecutive weekdays. We refer to improvements between the pretest and posttest on the trained condition as learning, and on any of the untrained conditions as generalization.

Conditions and stimuli. For all of the conditions, the task was to determine which of two pairs of tone pips was separated by a longer temporal interval (Fig. 1). On each trial, one of the pairs of tone pips, selected at random, was separated by a standard interval $(t)$, and the other was separated by a longer comparison interval $(t+\Delta t)$. Listeners indicated whether the comparison interval came first or second by pressing a key on a computer keyboard and were given visual feedback as to whether the response was correct or incorrect after every trial throughout the experiment. The response was not timed. We varied the $\Delta t$ adaptively across trials to determine the $\Delta t$ needed to achieve $79 \%$ correct performance (see Procedure).

The results for three temporal-interval discrimination conditions tested in the pretest and posttest are reported here. In the trained condition, the standard interval was $100 \mathrm{~ms}$, and the frequency of the tone pips was $1 \mathrm{kHz}(100 \mathrm{~ms}, 1 \mathrm{kHz})$. In the remaining two, untrained conditions, either the frequency ( $4 \mathrm{vs} 1 \mathrm{kHz} ; 100 \mathrm{~ms}, 4 \mathrm{kHz}$ ) or the interval (50 vs 100 $\mathrm{ms} ; 50 \mathrm{~ms}, 1 \mathrm{kHz}$ ) of the standard stimulus differed from the trained condition. We selected these untrained stimuli because of previous reports that learning on temporal-interval discrimination generalizes from the trained stimulus to an untrained stimulus frequency, but not to an untrained temporal interval — a pattern indicative of stimulus learningwith $10 \mathrm{~d}$ of practice (Wright et al., 1997; Karmarkar and Buonomano, 2003). The choice of these stimuli thus allowed us to examine the time course of improvement, or lack thereof, for an untrained stimulus to which learning was expected to generalize and to another to which learning was not expected to generalize with the longest training regimen we provided. We collected data on three other untrained conditions from each group, but those conditions differed across groups, so those results are omitted.

Each tone pip was presented at $86 \mathrm{~dB}$ SPL, had a total duration of 15 $\mathrm{ms}$, including $5 \mathrm{~ms}$ raised-cosine rise/fall ramps, and was presented in zero phase. The temporal interval between the two pips was measured from the onset of the first pip to the onset of the second pip. The onsets of the first pips of the first and second stimulus presentations were separated by $900 \mathrm{~ms}$. We generated the tones digitally using a digital signalprocessing board (TDT AP2, Tucker Davis Technologies), delivered them to a 16 bit digital-to-analog converter (TDT DD1), followed by an antialiasing filter $(8.5 \mathrm{kHz}$ low-pass; TDT FT5), an attenuator (TDT PA4), and a headphone buffer (TDT HB6), and then into the left earpiece of Sennheiser HD625 headphones with circumaural cushions.
Procedure. We measured temporal-interval discrimination thresholds using an adaptive, two-presentation, forced-choice procedure, with feedback. Within each 60 -trial block, we adaptively adjusted $\Delta t$ by decreasing it after every three consecutive correct responses, and increasing it after each incorrect response. The $\Delta t$ values at which the direction of change reversed from decreasing to increasing or vice versa are referred to as "reversals." After discarding the first three or four reversals from each block, ensuring that an even number of reversals remained, we calculated the average of the $\Delta t$ values of the remaining reversals to estimate the $79 \%$ correct point on the psychometric function, denoted as the threshold (Levitt, 1971). We estimated the threshold from a block of trials only if there were seven or more total reversals in the block. The initial $\Delta t$ in each block of trials was always $0 \mathrm{~ms}$, forcing the listener to guess. The step size was $10 \%$ of the standard interval through the third reversal, and $1 \%$ of the standard interval thereafter. Throughout this article, threshold is expressed as a Weber fraction, $\Delta t / t$. Thus, a threshold of 0.2 for a standard interval of $100 \mathrm{~ms}$ indicates that the listener could discriminate 120 ms from $100 \mathrm{~ms}$ on $79 \%$ of trials.

We collected five threshold estimates (300 trials) for each condition in the pretest and posttest, and completed testing on each condition before proceeding to the next. During these tests, the conditions were presented in random order across listeners, but the condition order of the pretest was always the same as that of the posttest for each individual listener. The pretest and posttest each took $\sim 2 \mathrm{~h}$, including brief breaks between conditions (approximately every $20 \mathrm{~min}$ ). During the training sessions, we collected 15 threshold estimates (900 trials) per day, taking $\sim 1 \mathrm{~h}$, including a brief break usually between the eighth and ninth threshold estimate.

Listeners. Twenty-seven paid participants (16 females) between the ages of 18 and 26 years served as listeners. These listeners practiced the trained condition for either $2 \mathrm{~d}$ ( $n=11$; new data), $4 \mathrm{~d}$ ( $n=10$; new data), or $10 \mathrm{~d}$ ( $n=6$; pretest and training phase data are from Wright and Sabin, 2007; posttest results new data). All listeners had normal hearing and had no prior experience with psychoacoustic tasks. One or more days before the pretest, all listeners attended a familiarization session in which they were given a basic hearing screening and introduced to the testing procedures using listening-in-noise conditions.

Some of the data were omitted from the final analyses. All of the results of one listener in the $2 \mathrm{~d}$ group were excluded because his posttest performance was aberrant, being worse than his pretest performance on four of the six conditions on which he was tested. For example, on the trained condition, his posttest threshold was more than 2 SDs higher than the group mean. All of the data of a $4 \mathrm{~d}$ listener were also excluded because she fell asleep several times during the training sessions. In addition, if an individual listener's pretest threshold on any given condition was more than 2 SDs higher than that of $>100$ listeners tested previously on that condition, we omitted all of that listener's data on that condition. Using this criterion, we removed the data of two listeners, one from the $2 \mathrm{~d}$ group and one from the $4 \mathrm{~d}$ group, on the trained condition (100 $\mathrm{ms}, 1 \mathrm{kHz}$ ) as well as of one listener from the $2 \mathrm{~d}$ group on the untrained frequency condition $(100 \mathrm{~ms}, 4 \mathrm{kHz})$. Removing the data of these two listeners from all of the conditions rather than only from the specific conditions on which their pretest thresholds were aberrant did not change the statistical conclusions reached from any of the analyses.

\section{Results}

The learning curves of the three groups followed a similar trajectory (Fig. 2). All three groups improved equivalently on the trained condition from the pretest through the second day of training according to a three-time (pretest, and training days 1 and 2) $\times$ three-group (2, 4 , and $10 \mathrm{~d}$ ) ANOVA, with repeated measures on time (time: $F_{(2,40)}=16.23 ; p<0.001$; group: $F_{(2,20)}=0.26 ; p=0.78$; time $\times$ group: $\left.F_{(2,40)}=0.24 ; p=0.91\right)$. The 4 and 10 d groups also improved equivalently from the pretest through the fourth day of training (time: $F_{(4,48)}=21.50 ; p<0.001$; group: $F_{(1,12)}<0.01 ; p=0.99$; time $\times$ group: $\left.F_{(4,48)}=0.91 ; p=0.47\right)$. Therefore, the performance on the untrained conditions by the three trained groups can be viewed as indicating the extent of generalization at each of three time points along a common learning curve. 


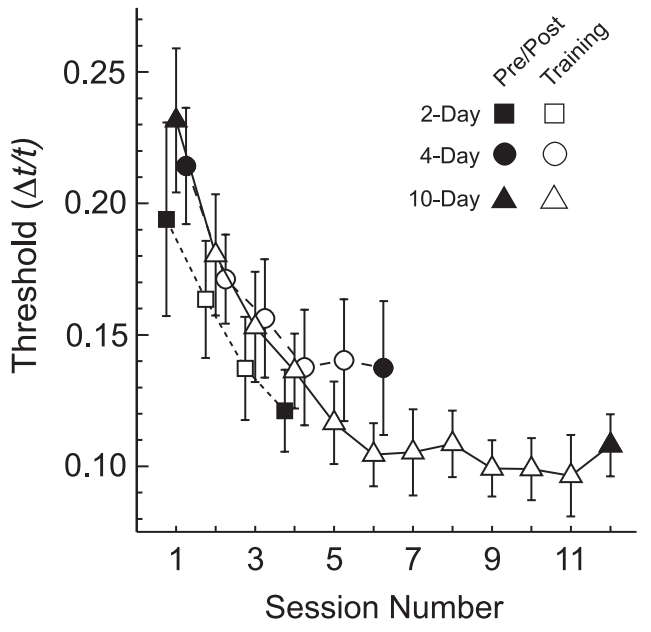

Figure 2. Learning curves: mean temporal-interval discrimination thresholds ( $\Delta t / t$ for $79 \%$ correct performance) on the trained condition ( $100 \mathrm{~ms}, 1 \mathrm{kHz}$ ). Results are shown for the pretest (Pre) and posttest (Post) (filled symbols) and for each of the training sessions (open symbols) for the listeners who received $2 \mathrm{~d}$ ( $n=9$; squares, short-dashed line), $4 \mathrm{~d}$ ( $n=8$; circles, long-dashed line), and $10 \mathrm{~d}$ ( $n=6$; triangles, solid line) of training. Error bars indicate \pm 1 SEM. All three groups improved between the pretest and posttest and did so following a similar trajectory.

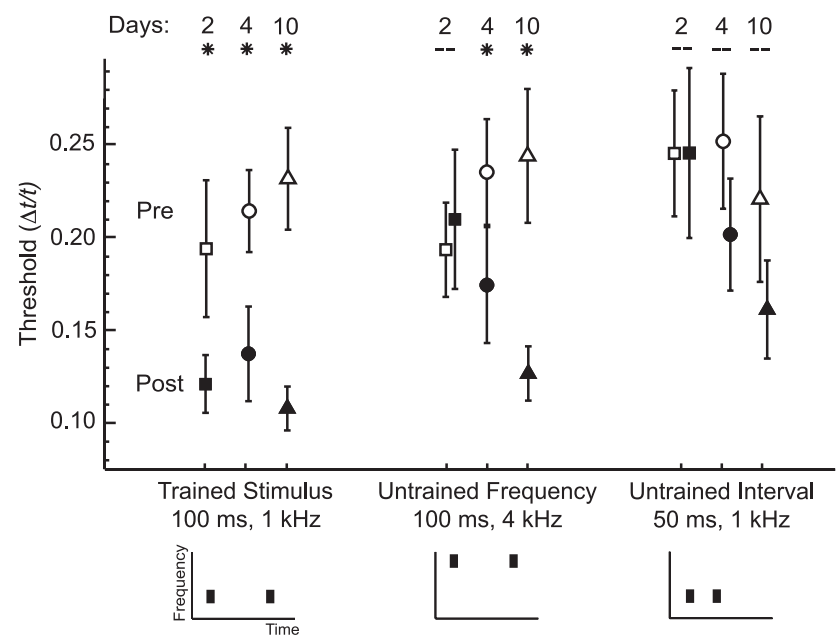

Figure 3. Generalization: mean temporal-interval discrimination thresholds ( $\Delta t / t$ for $79 \%$ correct performance) before (Pre; open symbols) and after (Post; filled symbols) training for the listeners who received $2 \mathrm{~d}$ ( $n=9-10$; squares), $4 \mathrm{~d}$ ( $n=8-9$; circles), and $10 \mathrm{~d}$ ( $n=6$; triangles) of training. Results are shown for the trained condition (100 ms, $1 \mathrm{kHz}$ ), the untrained-frequency condition (100 $\mathrm{ms}, 4 \mathrm{kHz})$, and the untrained-interval condition ( $50 \mathrm{~ms}, 1 \mathrm{kHz})$. Asterisks mark the cases in which the improvement between the pretest and posttest was statistically significant (all $p \leq 0.03$ ); dashes mark the remaining cases (all $p \geq 0.13$ ). Error bars indicate \pm 1 SEM. All three groups improved between the pretest and posttest on the trained condition, but only the 4 and $10 \mathrm{~d}$ groups generalized that learning to the untrained frequency.

The learning on the trained condition only generalized to the untrained stimulus frequency relatively late in the training phase, and never generalized to the untrained stimulus interval (Fig. 3). Between the pretest (open symbols) and posttest (filled symbols), all three trained groups showed significant learning of similar magnitude on the trained condition (100 ms, $1 \mathrm{kHz}$ (Fig. 3, left). A two-time (pretest and posttest $) \times$ three-group $(2,4$, and $10 \mathrm{~d})$ ANOVA with repeated measures on time revealed a significant main effect of time $\left(F_{(1,20)}=45.15 ; p<0.001\right)$ but no significant time $\times$ group interaction $\left(F_{(2,20)}=1.30 ; p=0.30\right)$. This learning was also confirmed in each group individually using paired subjects $t$ tests (all $p \leq 0.03$ ). In contrast, only the 4 and $10 \mathrm{~d}$ groups

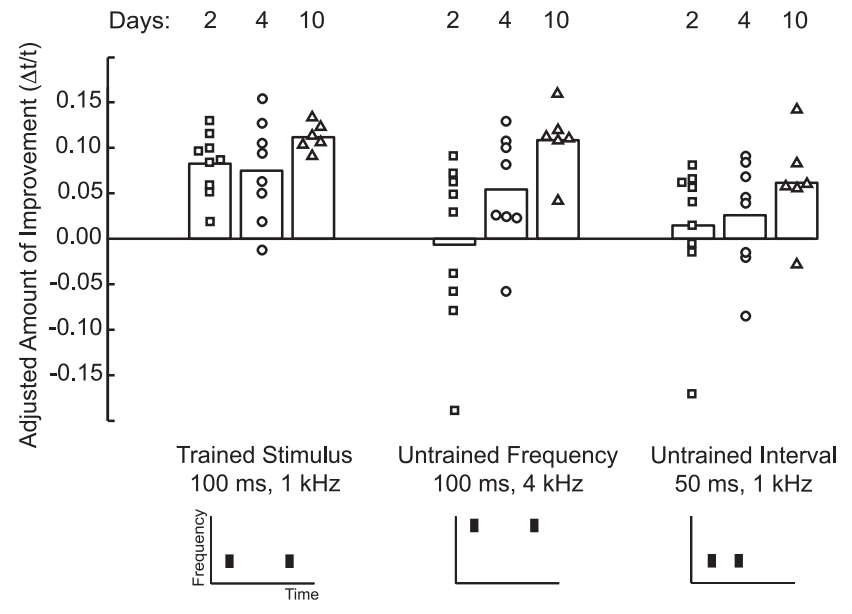

Figure 4. Amount of improvement of individual listeners: The amount of improvement of the individual listeners (symbols) who received $2 \mathrm{~d}$ (squares), $4 \mathrm{~d}$ (circles), and $10 \mathrm{~d}$ (triangles) of training, as well as of the mean of each group (bars). Results are shown for the trained condition (100 ms, 1 $\mathrm{kHz}$ ), the untrained-frequency condition $(100 \mathrm{~ms}, 4 \mathrm{kHz}$ ), and the untrained-interval condition ( $50 \mathrm{~ms}$, $1 \mathrm{kHz}$ ). The values represent the amount of improvement between the pretest and posttest for each listener, taking individual differences in starting threshold into account. These values were computed by adjusting the posttest threshold of each listener using the pretest threshold as a covariate, and then subtracting the individual adjusted posttest threshold from the pretest threshold averaged across all listeners on that condition. Nearly every listener improved (a positive value on the figure) on the trained condition, regardless of the number of days of training. In contrast, while all but one of the 4 and $10 \mathrm{~d}$ listeners generalized their learning on the trained condition to the untrained stimulus frequency, only approximately half of the $2 \mathrm{~d}$ listeners did.

generalized their learning to the untrained condition with a different frequency from, but the same standard interval as, the trained condition (100 ms, $4 \mathrm{kHz}$ ) (Fig. 3, middle). For this condition, there was a significant main effect of time $\left(F_{(1,21)}=13.17\right.$; $p=0.001)$ and a significant time $\times$ group interaction $\left(F_{(2,21)}=\right.$ $6.76 ; p=0.005)$. While the $2 \mathrm{~d}$ group did not improve ( $t$ test, $t_{(8)}$ $=-0.57 ; p=0.58)$, the groups who practiced more extensively did improve $\left(4 \mathrm{~d}: t_{(8)}=3.41 ; p=0.009 ; 10 \mathrm{~d}: t_{(5)}=4.33 ; p=\right.$ $0.008)$. Thus, the generalization to the untrained frequency lagged behind the learning on the trained condition.

None of the three trained groups generalized their learning to the untrained condition with a different standard interval from, but the same frequency as, the trained condition $(50 \mathrm{~ms}, 1 \mathrm{kHz})$ (Fig. 3, right). In this case, though the raw values of the posttest tended to decrease with increased training, there was no significant time $\times$ group interaction $\left(F_{(2,22)}=1.18 ; p=0.33\right)$. The main effect of time was borderline significant $\left(F_{(1,22)}=4.19 ; p=\right.$ $0.053)$, but, critically, none of the individual groups showed improvement ( $t$ tests, all $p \geq 0.13$ ). Therefore, if there was any generalization to this condition for any of the groups, its magnitude was small enough that it required greater statistical power to be observed than did the generalization to the untrained frequency. There were no significant main effects for group for any of the conditions (all $p \geq 0.59$ ). All of these statistical conclusions hold when posttest thresholds are compared across groups using pretest thresholds as a covariate (ANCOVA), thereby taking individual differences in initial performance into account.

Finally, the performance patterns identified in the group data are also evident at the individual level (Fig. 4). Nearly every listener (symbols) improved on the trained condition, regardless of the number of days of training (Fig. 4, left). In contrast, for the untrained stimulus frequency, while only approximately half of the $2 \mathrm{~d}$ listeners improved, all but one of the 4 and $10 \mathrm{~d}$ listeners did (Fig. 4, middle). It is noteworthy that though six of the nine 
$2 \mathrm{~d}$ listeners learned as much as the six $10 \mathrm{~d}$ listeners on the trained condition, the distributions of the improvement scores for these two groups were essentially nonoverlapping on the untrained-frequency condition. Individual performance for the untrained stimulus interval ranged from worsening in a few listeners to modest improvement in most, with comparable distributions across all three groups (Fig. 4, right). The amount of improvement on the untrained conditions was not significantly correlated with that on the trained condition for any of the three trained groups considered separately, though the correlation between the improvements on the untrained-interval condition and the trained condition approached significance for the $4 \mathrm{~d}$ group ( $r=0.69, p=0.058$; all others, $r<0.43, p>0.25)$. However, when the data of the 4 and 10 d groups were combined because both groups had shown generalization to the untrained frequency, there was a significant correlation in the amount of improvement between the untrained-frequency condition and the trained condition $(r=0.55, p=0.04)$, but not between the untrained-interval condition and the trained condition $(r=0.40$, $p=0.16)$ (also see Wright et al., 1997).

\section{Discussion}

The purpose of this investigation was to examine the relationship between the changes that underlie perceptual learning on a trained stimulus and its generalization to untrained stimuli by comparing the time courses of these two types of improvement on an auditory temporal-interval discrimination task. The idea was that a difference in these two time courses would indicate that different changes can underlie learning and generalization. The time courses did differ. The time course of learning on the trained stimulus $(100 \mathrm{~ms}, 1 \mathrm{kHz})$ was relatively rapid, with statistically significant improvement observed after only two daily 900 -trial training sessions (testing on the third day). However, the time course of generalization to the untrained stimulus frequency (100 $\mathrm{ms}, 4 \mathrm{kHz}$ ) was considerably longer, with improvements not appearing until after four training sessions (testing on the fifth day). Thus, generalization to the untrained frequency only emerged well after improvements on the trained stimulus were evident. There was no improvement on the untrained temporal interval $(50 \mathrm{~ms}, 1 \mathrm{kHz})$ after any of the three training amounts $(2,4$, or $10 \mathrm{~d}$ ). The different time courses of these improvements indicate that perceptual learning and generalization can arise from changes that are at least partially distinct. That the emergence of the generalization lagged behind that of the learning suggests that increased training could expand the array of untrained conditions to which learning generalizes (the breadth of generalization) on some perceptual tasks.

The present results appear to be unique in demonstrating that the breadth of generalization in human perceptual learning can increase with increased training. As mentioned in the Introduction, in what seems to be the only other direct investigation of the multiple-session time course of generalization in perceptual learning, learning generalized to untrained stimulus orientations on a visual pop-out detection task both early and late in training (Ahissar and Hochstein, 1996). It has also been noted (Karni and Sagi, 1993) that learning on a visual texture discrimination task generalized from the trained to the untrained eye at the end of a single training session in one investigation (Karni and Sagi, 1993) but not after 8-10 training sessions in another (Karni and Sagi, 1991). This comparison suggests that the learning actually became more specific to the trained eye with increased training, a pattern that is also consistent with the idea that different changes can underlie learning and generalization (for examples of in- creasing specificity with increased training in motor-sequence learning, see Hikosaka et al., 1999; Korman et al., 2003; Park and Shea, 2003, 2005). However, such across-experiment comparisons must be interpreted with caution because small procedural differences among investigations may have large effects on generalization. For example, in the case of visual texture discrimination, others have reported that there is generalization to the untrained eye with multiple-session training (Schoups and Orban, 1996), suggesting that the specificity of learning may not increase with increased training on this task.

In contrast to these other investigations, here the breadth of generalization increased over the course of training because generalization only emerged after multiple practice sessions. The only other example of delayed generalization of perceptual learning of which we are aware comes from an investigation of learning in honeybees. Bees who received 21 training trials discriminating between two visual patterns learned on the trained patterns but did not generalize their learning to simplified versions of those patterns, while bees who received 42 training trials learned on both stimulus sets (Stach and Giurfa, 2005). We also note another recent report of the influence of training on the ability of humans to identify isolated words in background noise. Participants improved on this task regardless of whether they practiced it for 5 or $12-15 \mathrm{~h}$, but the only indication that this learning generalized to lexically hard sentences occurred in participants who had received the more extensive training ( $5 \mathrm{~h}$ : 0 of 9 listeners; 12-15 h: 4 of 8 listeners) (Burk and Humes, 2007).

One potential explanation for the increase in the breadth of generalization with increased training in the current data comes from the idea that generalization is determined by the inherent tuning of the particular neural circuitry that is modified during training (e.g., Karni, 1996; Ahissar et al., 2009). From this perspective, the learning on temporal-interval discrimination ultimately was specific to the trained interval but generalized to an untrained frequency because extensive training modified circuitry that was tuned to the temporal but not the spectral aspects of these stimuli. This idea could account for the delayed generalization in the present results if the circuitry that was modified early in training differed from and was more narrowly tuned than that modified later in training. The possibility that the site of modification changed over the course of practice receives some support from reports that the brain regions involved in learning can differ at different time points during training (e.g., Karni et al., 1998; Petersen et al., 1998; Atienza et al., 2002; Gottselig et al., 2004; Takashima et al., 2007) (for review, see Kelly and Garavan, 2005). It is also consistent with a proposal that perceptual training leads to modifications in different neural processes depending on which one provides a signal-to-noise ratio sufficient for performing the trained task at a given time point in training (Ahissar et al., 2009). The delayed generalization observed here could also be accounted for in this inherent tuning framework if the same circuitry was modified throughout the course of training, but the training affected the extent to which the potential tuning of that circuitry was revealed. For example, the current training might have modified circuitry that initially responded only to a restricted stimulus set but that had some subthreshold sensitivity to a wider range of stimuli (e.g., Kaur et al., 2004). If so, generalization would have been delayed if the training first affected the responses of that circuitry to its originally favored stimuli and only later strengthened the responses to the wider range of stimuli as well, thereby ultimately revealing the full inherent tuning capacity of that circuitry.

Another potential explanation comes from the view that generalization reflects how input from more peripheral circuitry is 
weighted centrally (Mollon and Danilova, 1996; Dosher and Lu, 1998; Petrov et al., 2005). According to this idea, the generalization pattern that resulted from $10 \mathrm{~d}$ of training on temporalinterval discrimination arose because learning occurred within centralized circuitry that weighted more heavily those inputs that provided information about the temporal compared with the spectral aspects of the stimulus. This explanation could account for the broadening of the generalization pattern with increased training if the relative weights assigned to these two stimulus dimensions changed over the course of training, being relatively equal early on to favoring the temporal dimension in the end. By this account, training consistently affected the same circuitry, but over time altered how that circuitry weighted incoming information. Some recent evidence is consistent with this possibility. A quantitative model of learning that was based solely on the incremental reweighting of inputs through a Hebbian rule revealed systematic, task-dependent changes in the weights assigned to inputs tuned to different stimulus characteristics on a visual orientation-discrimination task (Petrov et al., 2005). In addition, physiologically, training-induced behavioral improvements in the discrimination of visual motion direction in monkeys were correlated with changes in neural responses in a brain region associated with decision making, but not in one associated with sensory encoding (Law and Gold, 2008). However, in that report, generalization was only assessed along a stimulus dimension for which generalization was expected to be minimal (from human data) and in fact was. Therefore, it is not clear whether the time course and characteristics of the physiological changes observed in that investigation would result in delayed generalization.

The present demonstration of delayed generalization also has practical significance. Because generalization maximizes the benefit received from practice, its induction is a goal of most practical applications of perceptual training. The current data illustrate that a training regimen that appears to be inadequate in this regard after a few training sessions may prove to be quite effective if the number of sessions is simply increased. An awareness of the potential for the late emergence of generalization therefore could aid the development and evaluation of practical perceptual-training schemes. For example, it could inform choices about the number of training sessions to be provided and influence appraisals of training effectiveness at different time points in training.

Together, the slower time course for generalization than learning that is documented here has theoretical, neurophysiological, and practical implications. From a theoretical perspective, these results suggest that learning and generalization are distinct forms of improvement that may differ on a range of characteristics in addition to their time courses. Different requirements for learning and generalization, such as training duration, may help to explain discrepancies as to the generalization associated with a given trained task. From a neurophysiological perspective, these data imply that the neural modifications that result from perceptual training either shift to a different locus or change in character as training progresses, thereby changing the range of stimuli that are affected by the modification. Finally, from a practical perspective, these data show that simply providing additional training can increase the number of untrained conditions to which learning generalizes. This offers a potential means to improve the efficacy of perceptual training used to treat disorders and enhance normal perceptual abilities.

\section{References}

Ahissar M, Hochstein S (1996) Learning pop-out detection: specificities to stimulus characteristics. Vision Res 36:3487-3500.
Ahissar M, Nahum M, Nelken I, Hochstein S (2009) Reverse hierarchies and sensory learning. Philos Trans R Soc Lond B Biol Sci 364:285-299.

Atienza M, Cantero JL, Dominguez-Marin E (2002) The time course of neural changes underlying auditory perceptual learning. Learn Mem 9:138-150.

Burk MH, Humes LE (2007) Effects of training on speech recognition performance in noise using lexically hard words. J Speech Lang Hear Res 50:25-40.

Dosher BA, Lu ZL (1998) Perceptual learning reflects external noise filtering and internal noise reduction through channel reweighting. Proc Natl Acad Sci U S A 95:13988-13993.

Gottselig JM, Brandeis D, Hofer-Tinguely G, Borbély AA, Achermann P (2004) Human central auditory plasticity associated with tone sequence learning. Learn Mem 11:162-171.

Hikosaka O, Nakahara H, Rand MK, Sakai K, Lu X, Nakamura K, Miyachi S, Doya K (1999) Parallel neural networks for learning sequential procedures. Trends Neurosci 22:464-471.

Karmarkar UR, Buonomano DV (2003) Temporal specificity of perceptual learning in an auditory discrimination task. Learn Mem 10:141-147.

Karni A (1996) The acquisition of perceptual and motor skills: a memory system in the adult human cortex. Brain Res Cogn Brain Res 5:39-48.

Karni A, Sagi D (1991) Where practice makes perfect in texture discrimination: evidence for primary visual cortex plasticity. Proc Natl Acad Sci U S A 88:4966-4970.

Karni A, Sagi D (1993) The time course of learning a visual skill. Nature 365:250-252.

Karni A, Meyer G, Rey-Hipolito C, Jezzard P, Adams MM, Turner R, Ungerleider LG (1998) The acquisition of skilled motor performance: fast and slow experience-driven changes in primary motor cortex. Proc Natl Acad Sci U S A 95:861-868.

Kaur S, Lazar R, Metherate R (2004) Intracortical pathways determine breadth of subthreshold frequency receptive fields in primary auditory cortex. J Neurophysiol 91:2551-2567.

Kelly AM, Garavan H (2005) Human functional neuroimaging of brain changes associated with practice. Cereb Cortex 15:1089-1102.

Korman M, Raz N, Flash T, Karni A (2003) Multiple shifts in the representation of a motor sequence during the acquisition of skilled performance. Proc Natl Acad Sci U S A 100:12492-12497.

Law CT, Gold JI (2008) Neural correlates of perceptual learning in a sensory-motor, but not a sensory, cortical area. Nat Neurosci 11:505-513.

Levitt H (1971) Transformed up-down methods in psychoacoustics. J Acoust Soc Am 49:467-477.

Mollon JD, Danilova MV (1996) Three remarks on perceptual learning. Spat Vis 10:51-58.

Ortiz JA, Wright BA (2009) Contributions of procedure and stimulus learning to early, rapid perceptual improvements. J Exp Psychol Hum Percept Perform 35:188-194.

Ortiz JA, Wright BA (2010) Differential rates of consolidation of conceptual and stimulus learning following training on an auditory skill. Exp Brain Res 201:441-451.

Park JH, Shea CH (2003) Effect of practice on effector independence. J Mot Behav 35:33-40.

Park JH, Shea CH (2005) Sequence learning: response structure and effector transfer. Q J Exp Psychol A 58:387-419.

Petersen SE, van Mier H, Fiez JA, Raichle ME (1998) The effects of practice on the functional anatomy of task performance. Proc Natl Acad Sci U S A 95:853-860.

Petrov AA, Dosher BA, Lu ZL (2005) The dynamics of perceptual learning: an incremental reweighting model. Psychol Rev 112:715-743.

Schoups AA, Orban GA (1996) Interocular transfer in perceptual learning of a pop-out discrimination task. Proc Natl Acad Sci U S A 93:7358-7362.

Stach S, Giurfa M (2005) The influence of training length on generalization of visual feature assemblies in honeybees. Behav Brain Res 161:8-17.

Takashima A, Nieuwenhuis IL, Rijpkema M, Petersson KM, Jensen O, Fernández G (2007) Memory trace stabilization leads to large-scale changes in the retrieval network: a functional MRI study on associative memory. Learn Mem 14:472-479.

Wright BA, Sabin AT (2007) Perceptual learning: how much daily training is enough? Exp Brain Res 180:727-736.

Wright BA, Buonomano DV, Mahncke HW, Merzenich MM (1997) Learning and generalization of auditory temporal-interval discrimination in humans. J Neurosci 17:3956-3963. 